\title{
Soft X-ray heating of the solar chromosphere during the gradual phase of two solar flares
}

\author{
A. Berlicki ${ }^{1,2}$ and P. Heinzel ${ }^{3}$ \\ 1 Observatoire de Paris, Section de Meudon, LESIA, 92195 Meudon Principal Cedex, France \\ 2 Astronomical Institute of the Wrocław University, ul. Kopernika 11, 51-622 Wrocław, Poland \\ 3 Astronomical Institute, Academy of Sciences of the Czech Republic, 25165 Ondrejov, The Czech Republic
}

Received 13 November 2003 / Accepted 2 February 2004

\begin{abstract}
In this paper we perform an analysis of the energetics of the solar flaring chromosphere irradiated by soft X-rays (SXR) emitted from overlying hot flare loops. To study an expected SXR heating we used the observations of two solar flares obtained with the Multichannel Subtractive Double Pass (MSDP) spectrograph attached to the Large Coronagraph of the Wrocław University. SXR observations of these flares come from the Yohkoh SXR telescope (SXT). From MSDP spectral images we derived mean $\mathrm{H} \alpha$ line profiles which were then used to construct semi-empirical non-LTE models of the chromosphere in the analyzed areas of flares. On the basis of SXR observations we calculated the mean emission measure $E M$ and the temperature in flaring coronal structures located above the $\mathrm{H} \alpha$ kernels under study and these two quantities were used to compute the SXR irradiation of the chromosphere between 1-300 ̊. For each flare model we then evaluated the energy deposit due to such irradiation and compared it with the net radiative cooling rates at all depths in the chromosphere. By means of such modelling we were able to demonstrate that the enhanced emission of selected $\mathrm{H} \alpha$ kernels observed during the gradual phase of these two solar flares cannot be explained in terms of the SXR heating of the chromosphere. We found that radiative losses in all layers of the chromosphere exceeded the amount of the energy deposited by the SXR radiation. However, for stronger flares with much enhanced $E M$ a certain contribution of SXR to the heating cannot be ruled out.
\end{abstract}

Key words. Sun: flares - Sun: chromosphere - line: profiles - Sun: X-rays, gamma rays

\section{Introduction}

A significant amount of the flare energy is radiated in soft $\mathrm{X}$-rays (SXR). Coronal flaring structures which emit this radiation are located above an active region and thus there is a possibility of radiative heating of the underlying chromosphere. Soft X-ray heating of the chromosphere by radiation coming from hot coronal structures was first proposed by Jefferies (1957) and Švestka (1957). The importance of this mechanism was also demonstrated by Somov (1975). He suggested that around bright compact features clearly seen in $\mathrm{H} \alpha$ images and heated probably by non-thermal electrons, there are diffuse and not so bright regions heated by SXR. Hénoux \& Nakagawa (1977) showed the effect of SXR heating for solar flares with a strong SXR emission originating in flaring loops during the flash phase. They have made detailed theoretical calculations of the rate of energy deposition from X-rays into the chromospheric layers. They concluded that soft X-ray radiation must be taken into account in interpreting the modification of the solar chromosphere during a flare and that the SXR heating predominates in the low temperature part of the chromosphere. Machado (1978) analyzed observational evidence of SXR

Send offprint requests to: A. Berlicki,

e-mail: arkadiusz.berlicki@obspm.fr heating in the upper and lower chromosphere and found that photons of the X-ray band 8-12 $\AA$ deposit their energy probably just in the $\mathrm{H} \alpha$ forming region. The SXR heating is now routinely included in flare modelling (Fang \& Hénoux 1983; Hawley \& Fisher 1992). In all published papers there is a general agreement that soft $\mathrm{X}$-ray radiation plays an important role in the heating of the solar chromosphere during the impulsive phases of solar flares. However, there is no detailed analysis of the SXR heating during the gradual phase based on observations of selected parts of the flaring structures performed in SXR as well as in some chromospheric lines (for example, in the $\mathrm{H} \alpha$ line).

In this paper we study the gradual phase of two solar flares. In our analysis we used the observations of these flares made in the $\mathrm{H} \alpha$ line and in SXR. The chromospheric parts of these flares were observed with Multichannel Subtractive Double Pass spectrograph (MSDP) coupled to the Large Coronagraph of the Wrocław University. To analyze the coronal structures of the flares we used the SXR images taken with the Soft X-ray Telescope (SXT) onboard the Yohkoh satellite. Analysis of the $\mathrm{H} \alpha$ spectral images taken with MSDP and SXR images recorded with Yohkoh SXT allows us to determine the rate of the SXR energy deposition in the chromosphere within selected 
parts of flare ribbons. We also use non-LTE modelling to find the energy budget in the analyzed parts of flares.

The paper is organized as follows. In Sect. 2 we present the observations of two analyzed flares obtained on 25 September 1997 and 21 June 2000. We used H $\alpha$ spectral images taken with Wrocław MSDP and Yohkoh SXT images. Section 3 provides a description of the methods used in the analysis of Yohkoh data as well as MSDP observations of the flares. We also determine for both flares the observational characteristics used in the further analysis. In Sect. 4 we describe the non-LTE codes used in our analysis and we present the resulting models of the chromosphere for the selected parts of flares. Section 5 briefly summarizes the basics of the method used in the calculation of the SXR energy deposition in the solar chromosphere and in Sect. 6 we describe the calculations of the radiative losses. In Sect. 7 we calculate the SXR energy deposition for the analyzed areas of two solar flares and compare it with the radiative losses determined in these areas. Finally, Sect. 8 summarizes our analysis and provides a discussion of our results.

\section{Flare observations}

In our analysis we used the observations of flares that occured in active regions located relatively close to the solar disk centre. In such a case we can easily compare the $\mathrm{H} \alpha$ chromospheric structures observed with the Wrocław MSDP with the hot coronal loops seen on Yohkoh SXT images. In our analysis it is important to make a precise coalignment between both $\mathrm{H} \alpha$ and SXR structures (see Sect. 3.2). $\mathrm{H} \alpha$ observations of the active regions of both flares were performed using the MSDP coupled to the Large Coronagraph and the Horizontal Telescope of the Wrocław University (Rompolt et al. 1993). The data were processed in a standard way (Mein 1991). The times of the MSDP observations indicated below correspond to the beginning of the appropriate scan and to take each scan we need about $50 \mathrm{~s}$. The spatial resolution of the obtained images was limited by the seeing, on average it was about 1 arcsec, but occasionally it was worse.

\subsection{Flare of 25 September 1997}

The C7.2 class solar flare was observed in the active region NOAA 8088. At 12:00 UT this active region was located roughly 29 degrees south the equator, passing across the central meridian. During the flare between 11:50:31 and 12:42:48 UT, we obtained 14 full scans ( $\mathrm{H} \alpha$ images) covering the decay phase of the flare. At 11:50:31 UT the first strong increase of the $\mathrm{H} \alpha$ emission was observed in an MSDP image. The $\mathrm{H} \alpha$ flare emission was concentrated mainly in seven brightenings (Fig. 1). Four of them were located in the eastern part of the active region close to the cluster of the following spots, FS. The other three flare brightenings were situated to the northeast of the leading spot, LS. The leading spot and the flare kernels $\mathrm{KA}, \mathrm{KB}, \mathrm{KC}$ and $\mathrm{KD}$ were located inside the area of negative-polarity magnetic field while the group of following spots and kernels KE, KF and KG were in the positive polarity magnetic field (see Fig. 1). A detailed description of the $\mathrm{H} \alpha$ evolution of the active region NOAA 8088 before the flare and

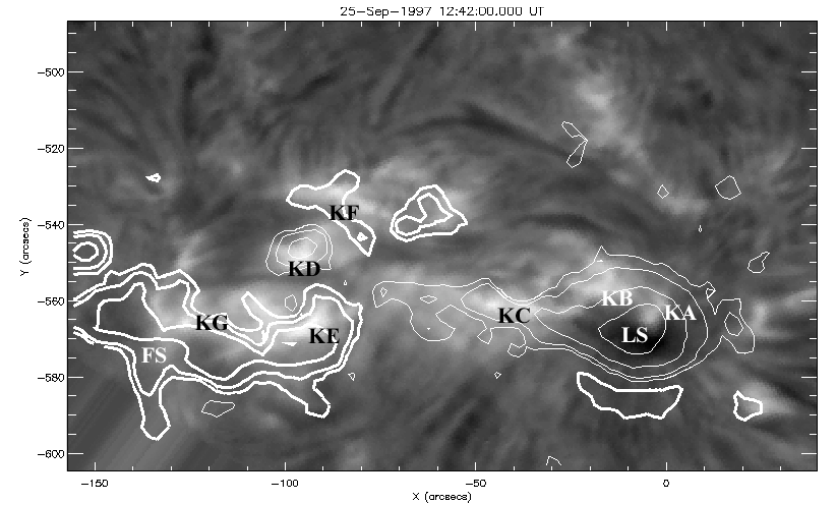

Fig. 1. Image of the flare of 25 September 1997 taken in the centre of the $\mathrm{H} \alpha$ line with MSDP spectrograph at 12:42 UT and overplotted by the SOHO/MDI (Michelson Doppler Imager) magnetogram taken at 08:00 UT. The thick white contours correspond to positive polarity magnetic field while the thin contours to negative polarity magnetic field. Labelled structures are described in the main text.

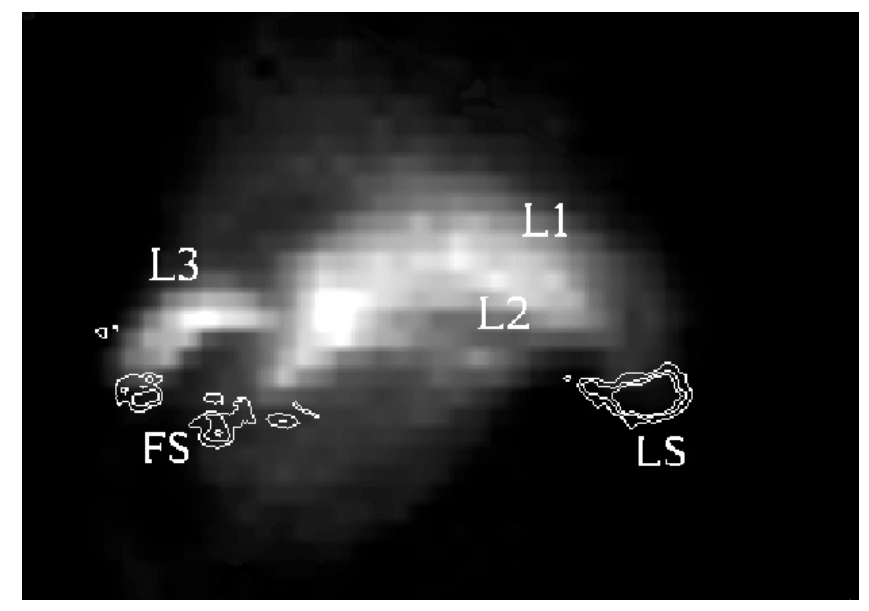

Fig. 2. Yohkoh SXT image of the loops recorded on 25 September 1997 at 12:38 UT through alluminium Al.1 filter and overplotted contours of the sunspots observed at 12:45 UT. Labelled structures are described in the main text.

development of the flare was presented by Falewicz \& Rudawy (1999). From the X-ray flux curve recorded by the GOES 9 satellite it follows that the flare started at 11:43 UT, reached its maximum at 11:49 UT and decayed until 14:00 UT. Images of the flare taken with the SXT in Al12 and Be119 filters show two big, parallel loops, L1 and L2, rooted between the leading spot and the eastern group of the following spots (Fig. 2). Another X-ray loop L3 was probably located below the L1 and L2 loops. Comparison of the $\mathrm{H} \alpha$ filtergrams with SXT images showed that footpoints of hot loops were situated close to the observed flare kernels. The loops L1 and L2 spanned the KA, KB and $\mathrm{KE} \mathrm{H} \alpha$ flare kernels, while the loop L3 spanned the kernels $\mathrm{KC}$ and KG (see also Fig. 1). In our analysis of the SXR heating we used five images (scans) taken at 12:05:52, 12:07:36, 12:09:25, 12:15:56 and 12:18:15 UT. 


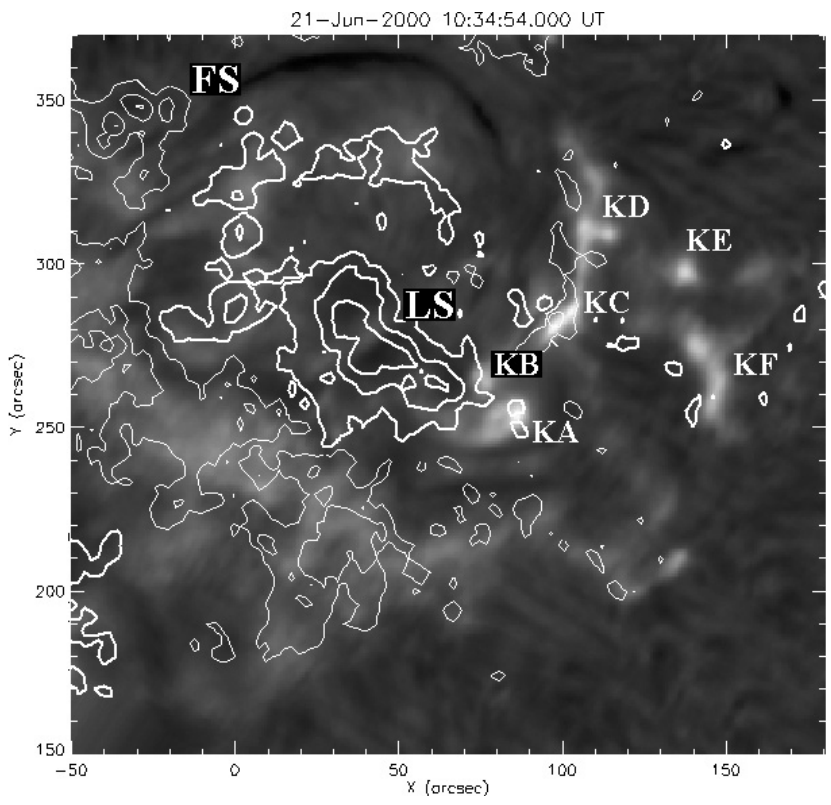

Fig. 3. MSDP image of the flare of 21 June 2000 taken in the $\mathrm{H} \alpha$ line centre at 10:34 UT and overplotted by the SOHO/MDI contours of magnetic fields taken at 11:12 UT. The thick and thin white contours correspond respectively to positive and negative polarity magnetic field. Labelled structures are described in the main text.

\subsection{Flare of 21 June 2000}

The C 4.5 flare was observed in the NOAA 9046 active region. At the time of the flare the active region was located about 20 degrees north of solar disk centre. $\mathrm{H} \alpha$ observations of this flare were performed with MSDP and the Large Coronagraph. During the flare between 10:11 and 11:00 UT several spectral MSDP images were taken. In our analysis we used two images made during the gradual phase of the flare at 10:46:08 and 10:50:56 UT. During this phase the $\mathrm{H} \alpha$ flare emission was concentrated mainly in several compact structures (Fig. 3). A few of them (KA, KB and KF) as well as leading sunspots (LS) were located within an area of positive polarity magnetic field. Emissions KC and KD were situated inside the area of negative polarity magnetic field. Analysis of the GOES 10 $\mathrm{X}$-ray flux curve showed that the flare onset occurred at about 10:10 UT, the maximum at 10:15 UT. After maximum the slow continuous decrease of X-ray emission was visible up to about 11:20 UT. SXT images of the flare taken with AlMg, Al12 and Be119 filters show at least six bright X-ray loops (Fig. 4). Two of them, spanning kernels $\mathrm{KA}, \mathrm{KB}$ and $\mathrm{KC}$ were very bright during the flare. Most of the flare kernels seen on $\mathrm{H} \alpha$ MSDP images were placed close to the footpoints of hot loops.

\section{Determination of parameters of the soft X-ray sources and observations of the $\mathrm{H} \alpha$ line profiles in analyzed areas of flares}

To estimate the rate of the energy deposited in the chromosphere by SXR radiation we first determined the parameters of SXR sources. Then, we used these parameters to calculate the spectral distribution of the intensity at the top of the chromosphere within the range 1-300 $\AA$. The SXR radiation deposits

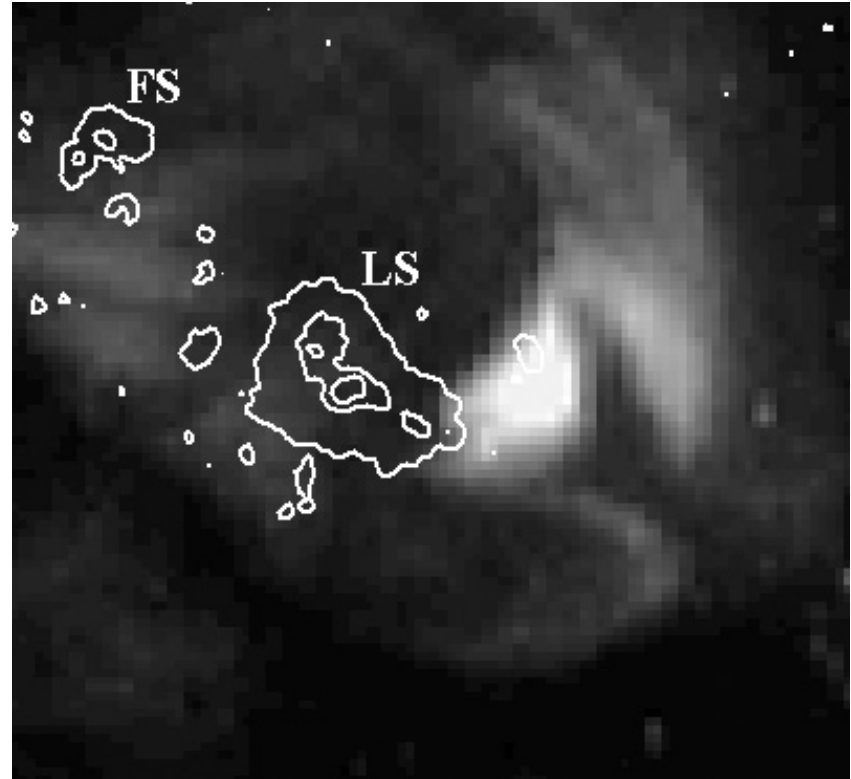

Fig. 4. Yohkoh SXT image of the loops observed during the flare of 21 June 2000 at 10:35 UT with the AlMg filter. The contours of the main sunspots observed at 12:45 UT are also presented. Labels LS and FS denote leading and following spots.

its energy in the solar chromosphere at different heights above the photosphere (defined by $\tau_{5000}=1$ ). The height distribution of deposited energy depends on the model of the chromosphere used in calculations. We used $\mathrm{H} \alpha$ line profiles obtained with the MSDP spectrograph to determine the models of the chromosphere in analyzed parts of flares (see Sect. 4).

\subsection{Parameters of the soft $X$-ray sources within analyzed parts of the flares}

The analysis of SXR heating was performed for some parts of the observed flares. For each flare we chose several areas where the enhanced $\mathrm{H} \alpha$ emission was observed as well as where the SXR structures were visible on SXT images. These areas also fulfilled the condition that the SXR emission of hot coronal structures should be located directly above the chromosphere where the enhanced $\mathrm{H} \alpha$ emission was observed and where the line profiles were recorded. Such a configuration often occurs in SXR loop footpoints and this configuration allowed us to use the plane-parallel geometry in the modelling of SXR heating.

For analysis of SXR heating of the chromosphere we chose three small areas A, B and C in the case of the flare of 25 September 1997 and five areas A-E for the flare of 21 June 2000. The position of these areas with respect to the SXR loops and chomospheric $\mathrm{H} \alpha$ structures for both flares is shown in Figs. 5 and 6. The contours of the squares on SXT images represent the dimension $A$ of the areas used in determination of the mean emission measure $E M$ and the temperature $T$. These parameters are given in Table 1 . In our calculations we assumed that the plasma inside each contour is homogeneous. The temperature and emission measure of plasma contained within selected areas were found from the SXT images by using the ratio of intensities in the Be119 and Al12 filters 

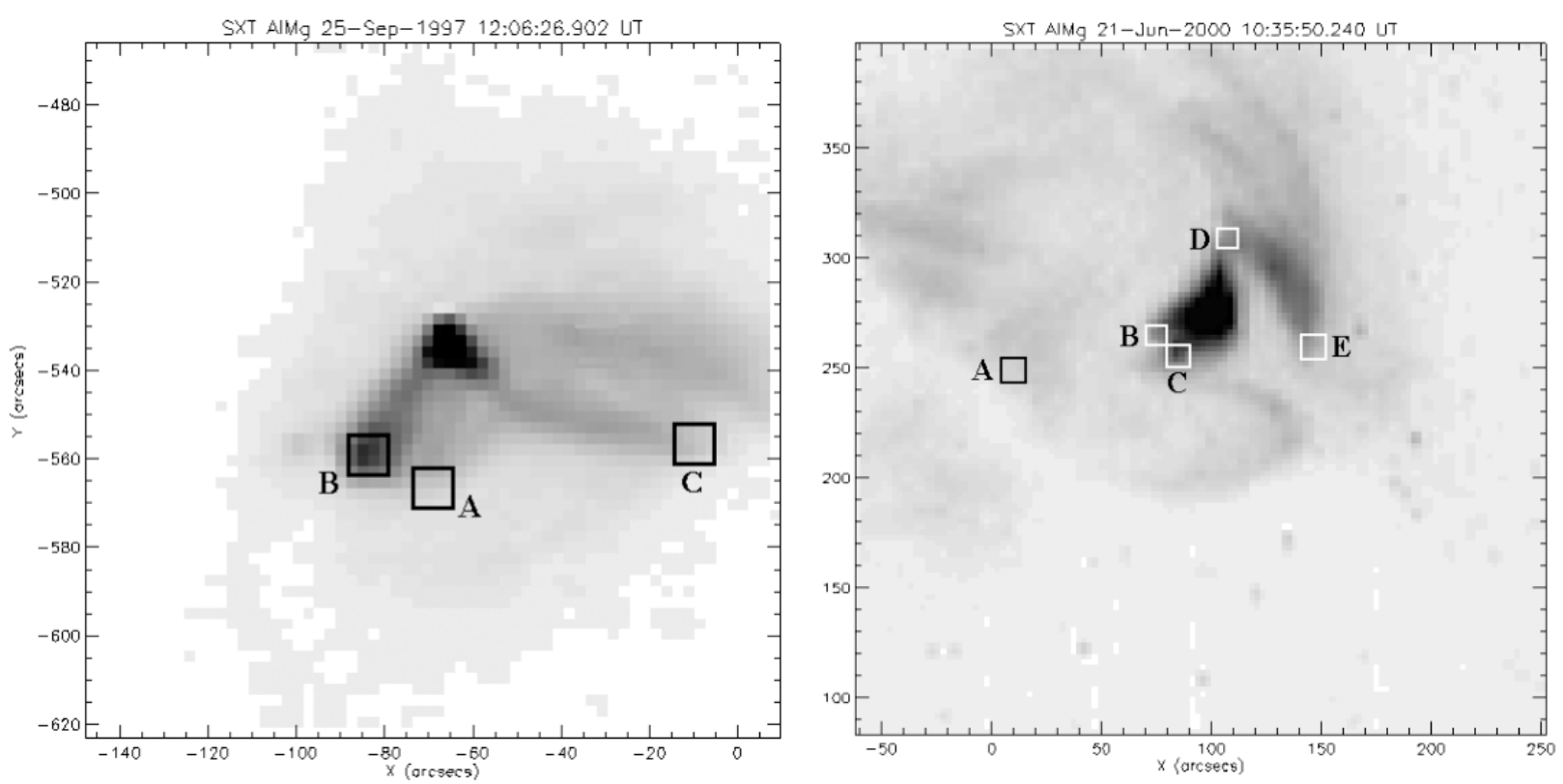

Fig. 5. Yohkoh SXT images of flaring structures of the flares on 25 September 1997 and 21 June 2000 with the contours of the areas used in the analysis of the SXR heating.
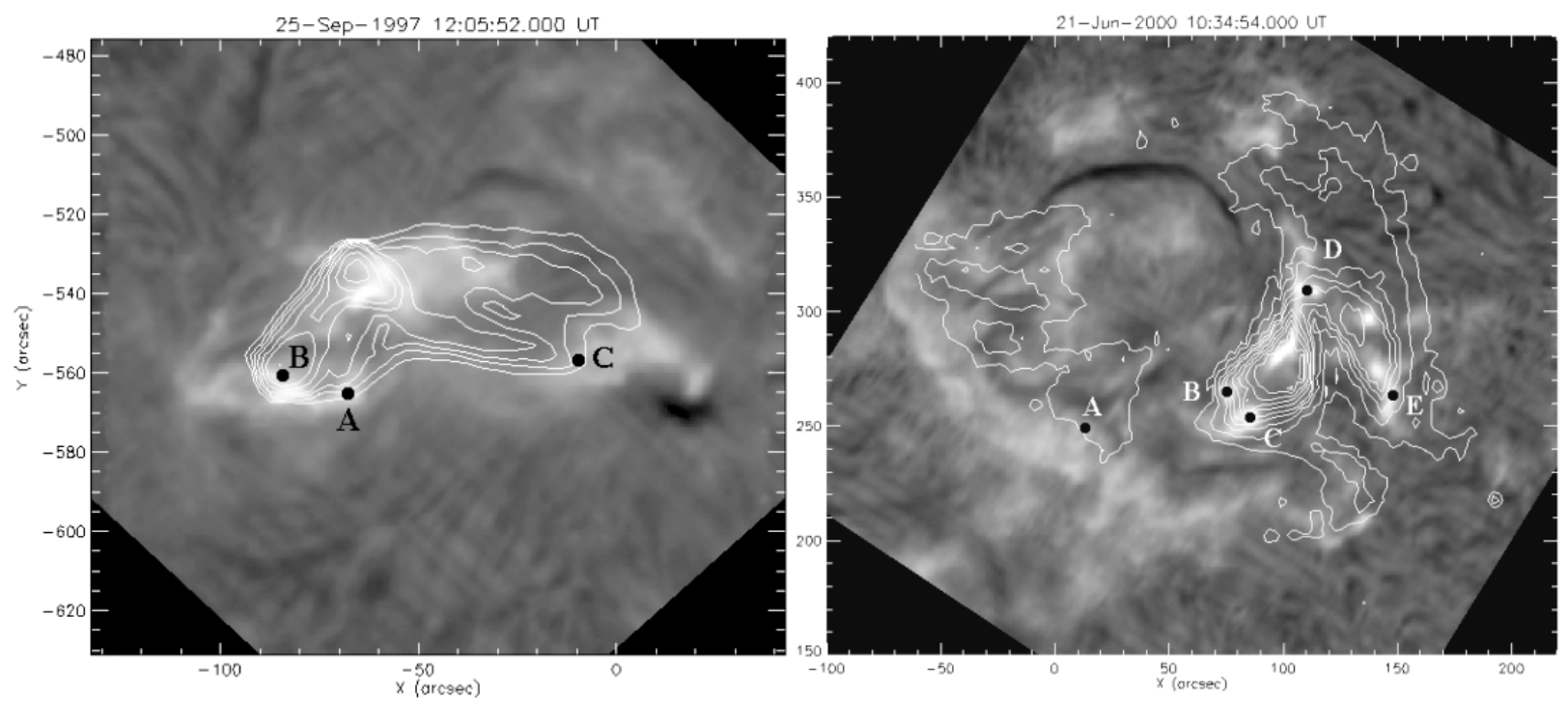

Fig. 6. Overlay of Yohkoh SXT flaring structures on the MSDP images taken during the gradual phase of two analyzed flares on 25 September 1997 and 21 June 2000. Black dots are the centres of the areas used in the analysis of the SXR heating.

(Tsuneta et al. 1991). We have used the standard procedures provided by the SolarSoft package. The accuracy of the determination of the temperature and emission measure of X-ray sources was of the order of $5 \%$.

We performed the analysis of SXR heating of the chromosphere for five moments of time during the gradual phase of the flare of 25 September 1997 and for two times for the flare of 21 June 2000. This limited number of time points results from the condition that the time difference between moments of determination of the parameters of the SXR sources and moments of observations of the $\mathrm{H} \alpha$ line profile should not be longer than a few minutes. This condition follows from characteristics of the gradual phase. During this phase the thermodynamical time-scale is rather long and we can assume that in a few minutes the parameters of the flare plasma do not change significantly. After inspection of the observations from the MSDP spectrograph and Yohkoh SXT it appears that there were only some pairs of the MSDP and SXT images that could be used in the analysis. Approximate times of these images are marked in Fig. 7 together with the plots of GOES 9 and 10 SXR fluxes for both flares.

\subsection{Ho line profiles observed in analyzed parts of the flares}

In our analysis it was very important to carry out detailed spatial co-alignment between MSDP and SXT images to 

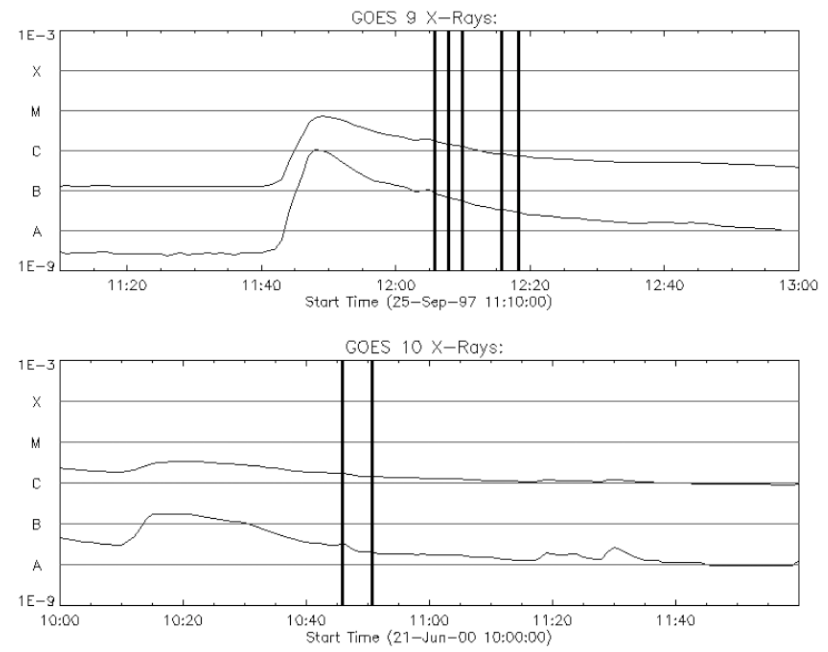

Fig. 7. Approximate times of the determination of parameters of SXT sources and the observations of $\mathrm{H} \alpha$ line profiles plotted against the GOES 9 and 10 SXR fluxes.

obtain $\mathrm{H} \alpha$ profiles in the same places where the parameters of SXR sources were determined. The main problem was the orientation of the MSDP $\mathrm{H} \alpha$ images. The peculiarities of the observations with the Wrocław MSDP cause the permanent rotation of the solar image in the focal plane. Additional difficulties follow from the instability of the coronagraph. The wind that can occur during the observations causes the shifts of the image in random directions. We have oriented the MSDP images using SOHO/MDI observations. On MSDP images made in the $\mathrm{H} \alpha$ line wings the sunspots were well visible, as on MDI. We used the sunspots as reference points and using spatiallycalibrated MDI images we have transformed the MSDP images to provide them with solar coordinates. The accuracy of co-alignment was of the order of $2^{\prime \prime}$ (the pixel-size of MDI images). On the spatially-oriented MSDP images we could estimate the position of the centres of given areas (see Fig. 5) used in the determination of the mean parameters of the hot plasma. The black dots seen on MSDP images (Fig. 6) are the centres of the corresponding areas. In these areas the mean $\mathrm{H} \alpha$ line profiles were obtained from the MSDP spectral images.

For each pixel of the spectral images the $\mathrm{H} \alpha$ line profiles are extracted automatically during the processing of MSDP data. In our analysis we obtained the mean profile for each analyzed area by averaging the profiles coming from nine pixels contained inside the $9 \operatorname{arcsec}^{2}$ square box. Then, the distance between two neighboring pixels was equal to 1 arcsec. The boxes from which the $\mathrm{H} \alpha$ line profiles were taken are much smaller than the size of the areas where the parameters of X-ray sources were obtained. This means that the heating of the chromosphere by SXR emission inside the boxes can be described in terms of a plane-parallel approximation.

Finally, we used $15 \mathrm{H} \alpha$ line profiles for the flare of 25 September 1997 (three areas at five different times) and 10 profiles for 21 June 2000 flare (five areas, two times). In Figs. 8 and 9 we present some profiles observed in analyzed parts of both flares. Obtained profiles are typical for the weak flare emission often observed during the late phase of solar
Table 1. The parameters of X-ray plasma obtained within the areas A, B and C for the flare on 25 September 1997 and in areas A-E for the flare on 21 June 2000. The appropriate areas are drawn in Fig. 5. In parentheses we present the times of MSDP images used for the determination of $\mathrm{H} \alpha$ line profiles.

\begin{tabular}{|c|c|c|c|c|}
\hline \multicolumn{5}{|c|}{25 SEPTEMBER 1997} \\
\hline Area & Time (UT) & $T(\mathrm{MK})$ & $E M\left(\mathrm{~cm}^{-3}\right)$ & $A\left(\mathrm{~cm}^{2}\right)$ \\
\hline \multirow{5}{*}{ A } & $12: 06: 26(12: 05: 52)$ & 7.5 & $5.0 \times 10^{46}$ & \multirow{5}{*}{$6.557 \times 10^{17}$} \\
\hline & $12: 07: 58(12: 07: 36)$ & 7.5 & $4.2 \times 10^{46}$ & \\
\hline & 12:09:54 (12:09:25) & 8.0 & $2.8 \times 10^{46}$ & \\
\hline & $12: 15: 56(12: 15: 56)$ & 7.0 & $3.2 \times 10^{46}$ & \\
\hline & $12: 16: 04(12: 18: 15)$ & 7.0 & $3.1 \times 10^{46}$ & \\
\hline \multirow{5}{*}{ B } & $12: 06: 26(12: 05: 52)$ & 7.0 & $3.7 \times 10^{47}$ & \multirow{5}{*}{$6.534 \times 10^{17}$} \\
\hline & $12: 07: 58(12: 07: 36)$ & 6.2 & $2.8 \times 10^{47}$ & \\
\hline & $12: 09: 54(12: 09: 25)$ & 6.4 & $1.5 \times 10^{47}$ & \\
\hline & $12: 15: 56(12: 15: 56)$ & 6.7 & $3.0 \times 10^{46}$ & \\
\hline & $12: 16: 04(12: 18: 15)$ & 7.0 & $2.0 \times 10^{46}$ & \\
\hline \multirow{5}{*}{$\mathrm{C}$} & $12: 06: 26(12: 05: 52)$ & 8.3 & $3.5 \times 10^{46}$ & \multirow{5}{*}{$6.459 \times 10^{17}$} \\
\hline & $12: 07: 58(12: 07: 36)$ & 7.3 & $4.0 \times 10^{46}$ & \\
\hline & 12:09:54 (12:09:25) & 7.0 & $4.8 \times 10^{46}$ & \\
\hline & $12: 15: 56(12: 15: 56)$ & 7.0 & $4.8 \times 10^{46}$ & \\
\hline & $12: 16: 04(12: 18: 15)$ & 7.2 & $4.7 \times 10^{46}$ & \\
\hline \multicolumn{5}{|c|}{21 JUNE 2000} \\
\hline \multirow[t]{2}{*}{$\bar{A}$} & $10: 46: 36(10: 46: 08)$ & 15.0 & $9.5 \times 10^{45}$ & \multirow[t]{2}{*}{$9.895 \times 10^{17}$} \\
\hline & $10: 49: 16(10: 50: 56)$ & 15.0 & $9.3 \times 10^{45}$ & \\
\hline \multirow[t]{2}{*}{ B } & $10: 46: 36(10: 46: 08)$ & 9.2 & $1.4 \times 10^{46}$ & \multirow[t]{2}{*}{$5.328 \times 10^{17}$} \\
\hline & $10: 49: 16(10: 50: 56)$ & 10.0 & $1.0 \times 10^{46}$ & \\
\hline \multirow[t]{2}{*}{$\mathrm{C}$} & $10: 46: 36(10: 46: 08)$ & 9.3 & $2.3 \times 10^{46}$ & \multirow[t]{2}{*}{$8.309 \times 10^{17}$} \\
\hline & $10: 49: 16(10: 50: 56)$ & 10.2 & $1.6 \times 10^{46}$ & \\
\hline \multirow[t]{2}{*}{$\mathrm{D}$} & $10: 46: 36(10: 46: 08)$ & 9.2 & $2.9 \times 10^{46}$ & \multirow[t]{2}{*}{$1.022 \times 10^{18}$} \\
\hline & $10: 49: 16(10: 50: 56)$ & 9.8 & $2.3 \times 10^{46}$ & \\
\hline \multirow[t]{2}{*}{$\mathrm{E}$} & $10: 46: 36(10: 46: 08)$ & 9.3 & $3.1 \times 10^{46}$ & \multirow[t]{2}{*}{$1.211 \times 10^{18}$} \\
\hline & $10: 49: 16(10: 50: 56)$ & 9.0 & $2.8 \times 10^{46}$ & \\
\hline
\end{tabular}

flares. These profiles are slightly asymmetrical which indicates the presence of evaporative features.

\section{Non-LTE modelling of the flaring chromosphere}

We used the $\mathrm{H} \alpha$ line profiles extracted from MSDP spectral images to find the models of the solar chromosphere within the analyzed areas of the flares. These observed line profiles were compared with the grid of synthetic $\mathrm{H} \alpha$ line profiles calculated by the non-LTE code developed by Heinzel (1995) and modified for flare conditions. This code allows us to find a new model of the solar flare chromosphere by modifying two input parameters and using the semiempirical model VAL-C of Vernazza et al. (1981) or the F1 weakflare model of Machado et al. (1980) as the starting ones. The code uses a 1D plane-parallel geometry and the atmosphere is in hydrostatic equilibrium. We analyzed flares during their gradual phase so we can neglect weak macroscopic velocity fields. Hydrogen excitation and the ionization equilibrium has been computed by solving simultaneously the radiative transfer equations, the equations of statistical equilibrium for five-level 

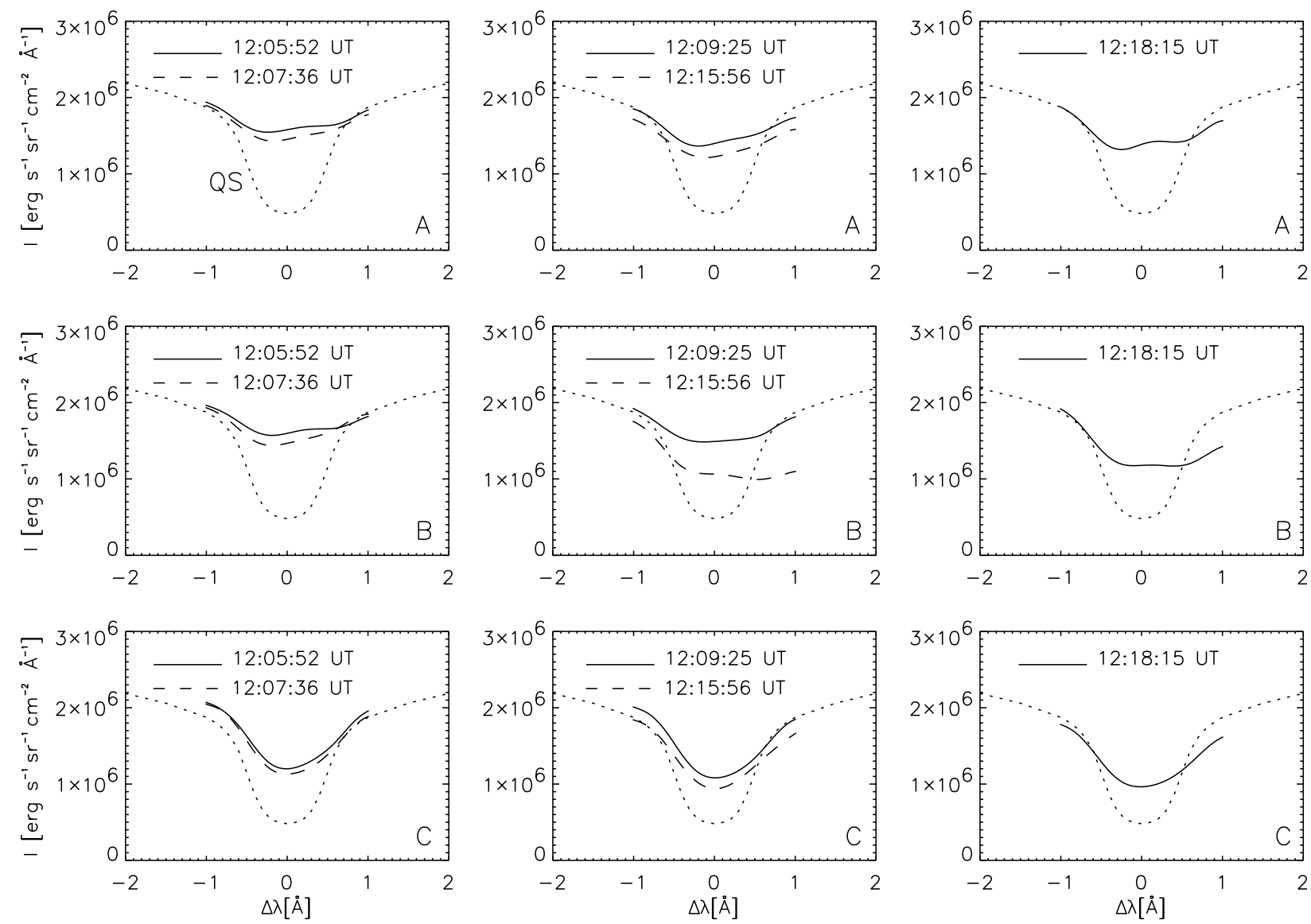

Fig. 8. The mean $\mathrm{H} \alpha$ line profiles observed within areas A, B and C during the flare on 25 September 1997 (solid and dashed lines). Dotted lines represent the mean $\mathrm{H} \alpha$ line profile emitted by the quiet Sun atmosphere in the vicinity of the flare.
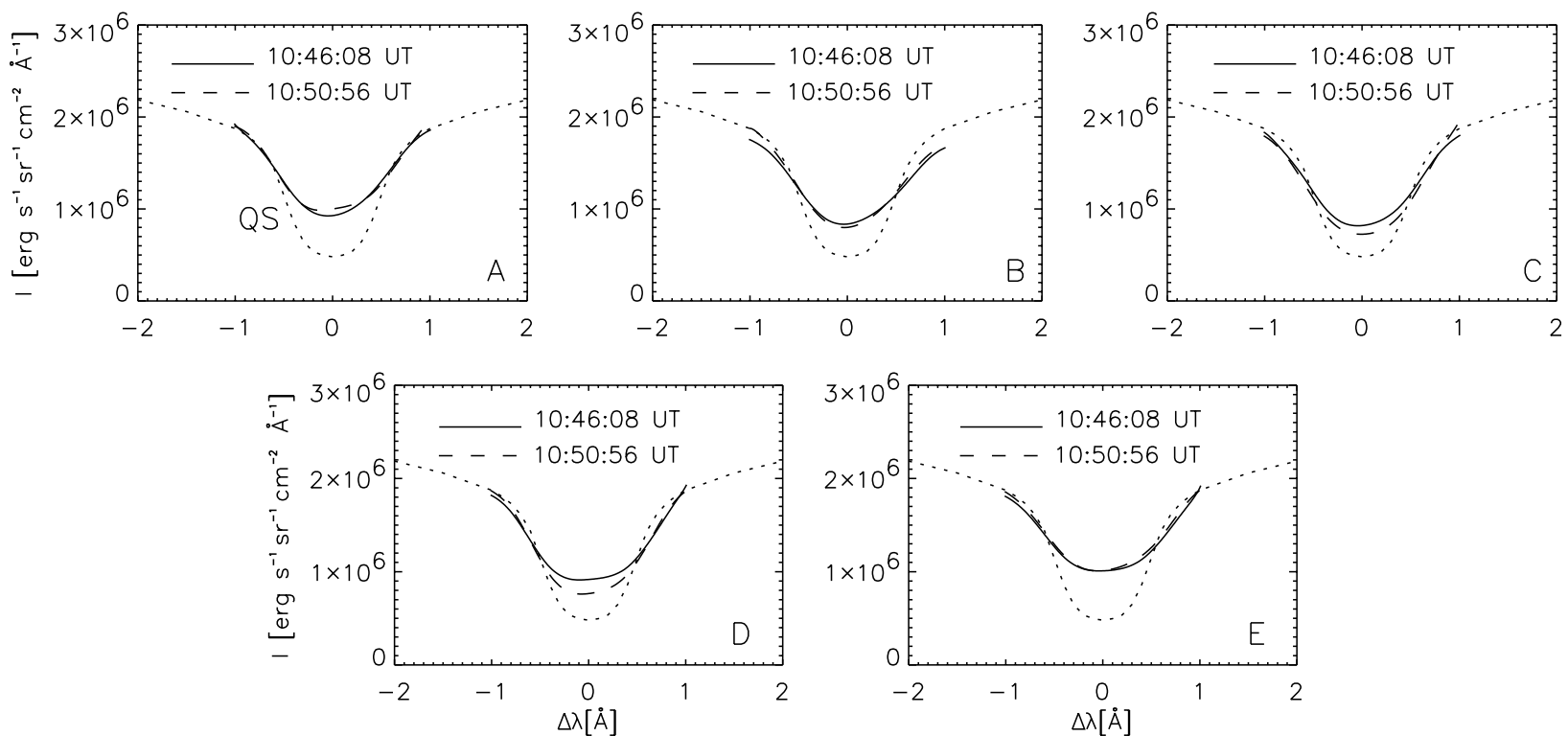

Fig. 9. The mean $\mathrm{H} \alpha$ line profiles observed within areas A, B, C, D and E during the flare on 21 June 2000 (solid and dashed lines). Dotted lines represent the mean $\mathrm{H} \alpha$ line profile emitted by the quiet Sun atmosphere in the vicinity of the flare.

plus the continuum atomic model of hydrogen and the equation of particle and charge conservation. The equations of statistical equilibrium have been preconditioned according to Rybicki \& Hummer (1991). The preconditioning is based on the lambda operator splitting technique, where the exact lambda operator is expressed as an approximate operator plus the correction. Then the correction is computed iteratively by the so-called Accelerated Lambda Iteration (ALI) method which is much 
faster and more reliable compared to standard Lambda iterations. This method is referred to as the MALI (Multilevel Accelerated Lambda Iterations) method. The preconditioned equations are then linearized with respect to electron density and atomic level populations and solved iteratively (Heinzel 1995). As input parameters used for modification of the starting (reference) atmospheric model we chose $m_{0}$ - modification of the $m$-scale in the model atmosphere, and then:

$m(I D)=m_{\mathrm{rm}}(I D)+m_{0}$,

and $\Delta T$ - parameter, which modifies the temperature distribution of the reference atmosphere so that a new temperature scale is defined by:

$T(I D)=T_{\mathrm{rm}}(I D)+\frac{\left[m_{1}-m(I D)\right] \Delta T}{m_{1}}$.

The subscript $\mathrm{rm}$ is related to the reference model (VAL-C or F1). Parameter $m_{1}$ determines the depth above which the temperature is modified (empirically we took $m_{1}=0.3$ ). $I D$ are the numbers of the layers in semiempirical models. Finally, we have computed the emergent profiles of the $\mathrm{H} \alpha$ line, taking into account the microturbulent and Stark broadening effects. In order to fit the observed spectra of the flare chromosphere we have constructed a grid of 206 semiempirical models. 150 of them have been created by modifying the VAL-C semiempirical model of the quiet Sun (QS) chromosphere, and 56 models have been calculated by using the F1 semiempirical model of a weak flare chromosphere as a starting model. For each model we have obtained the synthetic profile of the $\mathrm{H} \alpha$ line. In our calculation we use a mean value of the direction cosine equal to 0.9 for both analyzed flares. Finally, we obtained 206 synthetic profiles of the $\mathrm{H} \alpha$ line. Next, we convolved all these profiles with the Gaussian function of half-width $0.28 \AA$ (equivalent to $13 \mathrm{~km} \mathrm{~s}^{-1}$ at the $\mathrm{H} \alpha$ line wavelength). This value was found empirically. The aim of this procedure was to simulate the macroturbulent broadening. Finally, for each $\mathrm{H} \alpha$ profile observed in analyzed areas of flares, we found the best fit among the synthetic profiles. In the fitting procedure we found the differences between given observed profile and all 206 synthetic profiles in 100 lambda-points located between -0.5 and $+0.5 \AA$ from the $\mathrm{H} \alpha$ line centre. Then, for all synthetic profiles we calculated the sum of squares of all differences and we found the synthetic profile for which this sum has the smallest value. In this way, we determined the models of the chromosphere within all analyzed areas at all times. The parameters of these models together with reference models are given in Table 2.

In Fig. 10 we present the temperature distribution vs. height for models found for selected analyzed areas. Analysis of the observed $\mathrm{H} \alpha$ line profiles and obtained models allow us to conclude that the structure of the chromosphere within flare ribbons changes during the considered range of time. For most of the areas we noticed the slow decrease of the $\mathrm{H} \alpha$ emission which indicates that the models of the flaring chromosphere approach that of the quiet Sun chromosphere.

We used these models in our calculations of the energy deposited in the chromosphere by SXR emission and in analysis of the net radiative cooling rates (NRCR).
Table 2. The parameters $m_{0}$ and $\Delta T$ of the models of the chromosphere within analyzed areas of flares deduced from the comparison of synthetic $\mathrm{H} \alpha$ line profiles with observed ones. VAL-C and F1 denote the reference models used in calculations.

\begin{tabular}{|c|c|c|c|c|}
\hline \multicolumn{5}{|c|}{25 SEPTEMBER 1997} \\
\hline Area & Time (UT) & $\operatorname{Model}_{0}$ & $m_{0}\left(\mathrm{~g} / \mathrm{cm}^{2}\right)$ & $\Delta T(\mathrm{~K})$ \\
\hline \multirow{5}{*}{ A } & $12: 05: 52$ & $\mathrm{~F} 1$ & $1.9 \times 10^{-4}$ & -150 \\
\hline & $12: 07: 36$ & $\mathrm{~F} 1$ & $1.9 \times 10^{-4}$ & -300 \\
\hline & $12: 09: 25$ & $\mathrm{~F} 1$ & 0.0 & 0 \\
\hline & $12: 15: 56$ & $\mathrm{~F} 1$ & 0.0 & -150 \\
\hline & $12: 18: 15$ & $\mathrm{~F} 1$ & 0.0 & 0 \\
\hline \multirow{5}{*}{ B } & $12: 05: 52$ & $\mathrm{~F} 1$ & $1.9 \times 10^{-4}$ & -150 \\
\hline & $12: 07: 36$ & $\mathrm{~F} 1$ & $1.9 \times 10^{-4}$ & -300 \\
\hline & $12: 09: 25$ & $\mathrm{~F} 1$ & $1.9 \times 10^{-4}$ & -300 \\
\hline & $12: 15: 56$ & $\mathrm{~F} 1$ & $-5.0 \times 10^{-5}$ & -300 \\
\hline & $12: 18: 15$ & $\mathrm{~F} 1$ & 0.0 & -300 \\
\hline \multirow{5}{*}{$\mathrm{C}$} & $12: 05: 52$ & $\mathrm{~F} 1$ & $-5.0 \times 10^{-5}$ & 0 \\
\hline & $12: 07: 36$ & $\mathrm{~F} 1$ & $-5.0 \times 10^{-5}$ & -150 \\
\hline & $12: 09: 25$ & $\mathrm{~F} 1$ & $-1.0 \times 10^{-4}$ & 0 \\
\hline & $12: 15: 56$ & VAL-C & $2.5 \times 10^{-4}$ & 1200 \\
\hline & $12: 18: 15$ & $\mathrm{~F} 1$ & $-1.0 \times 10^{-4}$ & -300 \\
\hline \multicolumn{5}{|c|}{21 JUNE 2000} \\
\hline \multirow[t]{2}{*}{$\mathrm{A}$} & $10: 46: 08$ & F1 & $-1.0 \times 10^{-4}$ & -300 \\
\hline & $10: 50: 56$ & $\mathrm{~F} 1$ & $-1.0 \times 10^{-4}$ & -150 \\
\hline \multirow[t]{2}{*}{ B } & $10: 46: 08$ & VAL-C & $3.2 \times 10^{-4}$ & 400 \\
\hline & $10: 50: 56$ & VAL-C & $2.5 \times 10^{-4}$ & 800 \\
\hline \multirow[t]{2}{*}{$\mathrm{C}$} & $10: 46: 08$ & VAL-C & $3.2 \times 10^{-4}$ & 200 \\
\hline & $10: 50: 56$ & VAL-C & $2.5 \times 10^{-4}$ & 0 \\
\hline \multirow[t]{2}{*}{ D } & $10: 46: 08$ & VAL-C & $3.2 \times 10^{-4}$ & 800 \\
\hline & $10: 50: 56$ & VAL-C & $2.5 \times 10^{-4}$ & 400 \\
\hline \multirow[t]{2}{*}{$\mathrm{E}$} & $10: 46: 08$ & $\mathrm{~F} 1$ & $-1.0 \times 10^{-4}$ & -150 \\
\hline & $10: 50: 56$ & F1 & $-1.0 \times 10^{-4}$ & -150 \\
\hline
\end{tabular}

\section{X-ray heating of the chromosphere}

In the evaluation of SXR energy deposit we need to calculate the rate of energy conversion from the SXR flux of wavelength $\lambda$ to photoelectrons (Hénoux \& Nakagawa 1977):

$\frac{\mathrm{d} E_{\lambda}^{P}}{\mathrm{~d} t}=\sum_{i} \frac{\mathrm{d} N_{i, \lambda}^{P}}{\mathrm{~d} t} E_{i}$,

where we denoted the rate of photoionization of the $i$ th element by $\mathrm{d} N_{i, \lambda}^{P} / \mathrm{d} t$ and $E_{i}=h v-\chi_{i}$ is the energy of photoelectron with $\chi_{i}$ being the ionization potential of the $i$ th element. In our calculations we used the approximation of a plane-parallel atmosphere so the equation of transfer reduces to

$\mu \frac{\mathrm{d} I_{\lambda}(z, \mu)}{\mathrm{d} z}=-N_{\mathrm{H}} \sigma I_{\lambda}(z, \mu)$,

where $I_{\lambda}$ is the specific intensity of SXR radiation at wavelength $\lambda, \mu$ is the cosine of the angle between the direction of photon propagation and the vertical $z, N_{\mathrm{H}}$ the total hydrogen density and $\sigma$ the total ionization cross-section

$\sigma=\sum_{i \neq \mathrm{H}} \frac{N_{i}}{N_{\mathrm{H}}} \sigma_{i}+(1-x) \sigma_{\mathrm{H}}$ 

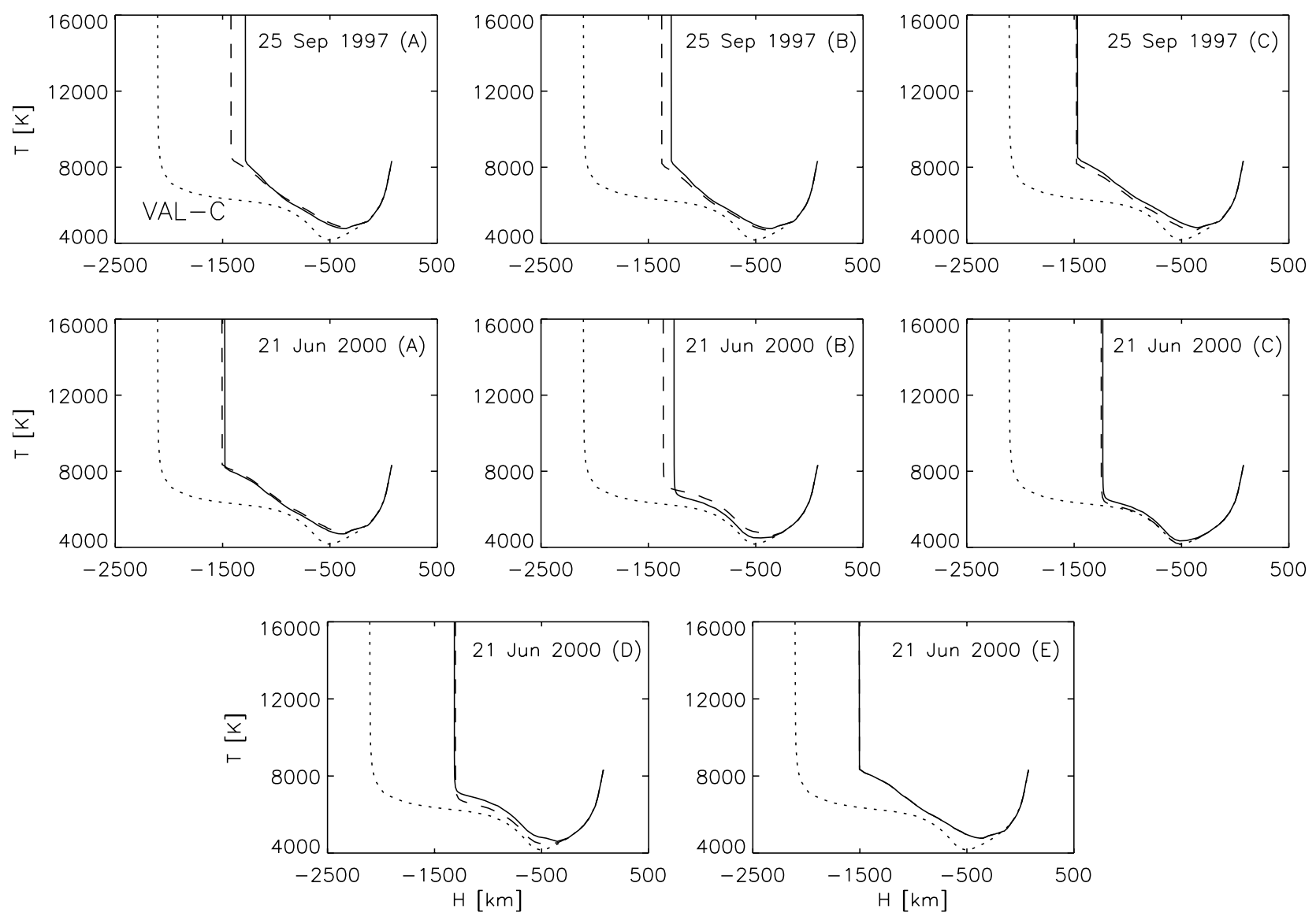

Fig. 10. Temperature as a function of height in models of the solar chromosphere obtained for analyzed areas of the flares. For the flare of 25 September 1997 the temperature is plotted for all areas (A, B and C) at 12:05:52 UT (solid lines) and at 12:18:15 UT (long-dashed lines) and for the flare of 21 June 2000 we made plot for all areas (A, B, C, D and E) at 10:46:08 UT (solid lines) and at 10:50:56 UT (long-dashed lines). For comparison we also plot the temperature distribution for VAL-C model of the quiet Sun (dotted line). Positive values of $h$ are at the bottom of the atmosphere.

The values of ionization cross-sections $\sigma_{i}$ for different elements were taken from Brown \& Gould (1970). The formal solution of the transfer equation is

$I_{\lambda}(z, \mu)=I_{\lambda_{0}}(\mu) \exp \left(\frac{m(z) \bar{\sigma}(z)}{\mu \rho}\right)$,

where we introduced the column mass $m$ defined as:

$$
m(z)=\int_{Z_{0}}^{Z} N_{\mathrm{H}}(z) \rho(z) \mathrm{d} z
$$

$I_{\lambda_{0}}(\mu)$ is the intensity of the SXR at the top of the atmosphere. We assumed this intensity to be uniform. $\rho$ is the mean molecular weight and $\bar{\sigma}$ is the effective ionization cross-section. After some substitutions and integration (see Hénoux \& Nakagawa 1977) we finally obtain:

$$
\frac{\mathrm{d} E_{\lambda_{1}, \lambda_{2}}^{P}}{\mathrm{~d} t}=2 \pi N_{\mathrm{H}} \int_{\lambda_{1}}^{\lambda_{2}} I_{\lambda_{0}} a(\lambda, z) E_{2}\left(\frac{m(z) \bar{\sigma}}{\rho}\right) \mathrm{d} \lambda .
$$

$E_{2}$ is is the exponential integral and $a(\lambda, z)$ is defined by:

$$
a(\lambda, z)=\sum_{i \neq \mathrm{H}} \frac{N_{i}}{N_{\mathrm{H}}} \sigma_{i}\left(1-\frac{X_{i}}{h v}\right)+\sigma_{\mathrm{H}}\left(1-\frac{X_{\mathrm{H}}}{h v}\right)(1-x) .
$$

Equation (8) allows us to calculate the energy deposit (in erg $\mathrm{cm}^{-3} \mathrm{~s}^{-1}$ ) at the specific height in the atmosphere.

To calculate the energy deposit we need to determine SXR intensity $I_{\lambda_{0}}$ at the top of the atmosphere within the spectral range we use. This intensity depends on the geometry, temperature structure and emission measure of the X-ray source. In the simplest case we can assume that at the top of the atmosphere the radiation is uniform. For an isothermal X-ray source of given temperature $T$ and emission measure $E M$ the power at wavelength $\lambda$ is $P_{\lambda}=E M \times \varepsilon(\lambda, T)$, where $\varepsilon(\lambda, T)$ is the emissivity of optically thin plasma. We use emissivities within the 1-300 A spectral range calculated by the SolarSoft package according to Mewe et al. (1985) and Mewe et al. (1986).

In the case of a plane-parallel approximation the emergent intensity in the direction normal to the atmosphere at wavelength $\lambda$ is:

$I_{\lambda}=\frac{P_{\lambda}}{4 \pi A}=\frac{E M \times \varepsilon(\lambda, T)}{4 \pi A}$,

where $A$ is the area of the X-ray source in projection on the plane of the atmosphere. To calculate the SXR energy deposit in the chromosphere we have used FORTRAN procedures written by us which allow us to determine the height distribution of the deposited energy. These procedures first calculate the mean 

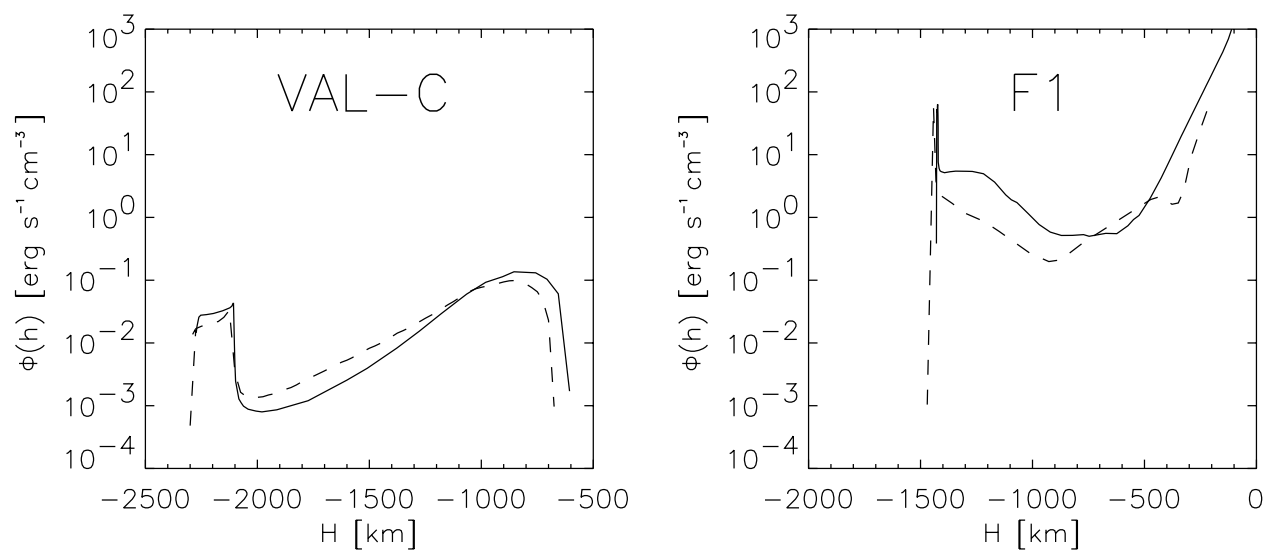

Fig. 11. The comparison of the NRCR for VAL-C and F1 semiempirical models calculated using our procedures (continuous lines) with the NRCR obtained by Vernazza et al. (1981) and Avrett et al. (1986) for these models (dashed lines).

intensity of SXR radiation reaching the top of the model chromosphere and then, using a given model of the chromosphere, the deposited energy.

\section{Evaluation of the net radiative cooling rates}

The amount of energy deposited in different layers of the chromosphere by SXR radiation can be compared with the net radiative cooling rates (NRCR) of the flare chromosphere. NRCR or radiative losses $\Phi_{i j}$ and $\Phi_{m}\left(\mathrm{erg} \mathrm{cm}^{-3} \mathrm{~s}^{-1}\right)$ can be calculated as a function of depth for line transitions $i \rightarrow j$ and for continua, respectively (see e.g. Vernazza et al. 1981 or Avrett et al. 1986). Assuming a steady-state, the sum of these rates must balance other energy inputs/outputs at each depth of the atmosphere. In general, this is described by a proper form of the energy-balance equation. For optically-thin plasma (solar corona and transition region), the NRCR are not coupled by the radiation transfer and they can be obtained directly from:

$\Phi=n_{\mathrm{e}} n_{\mathrm{H}} Q(T)$,

where $n_{\mathrm{e}}$ and $n_{\mathrm{H}}$ denote the electron and hydrogen density, respectively, and $Q(T)$ is the radiative loss function (Cox \& Tucker 1969; Raymond \& Smith 1977; Rosner et al. 1978). However, in the lower atmosphere this formula is no longer valid because the plasma becomes optically thick in some transitions.

In this case it is necessary to use a more complex equation which has the general form:

$\Phi=4 \pi \int_{0}^{\infty} \kappa_{v}\left(S_{v}-J_{v}\right) \mathrm{d} v$.

$S_{v}$ is the source function, $J_{v}$ is the mean intensity of the radiation field and $\kappa_{v}$ is the absorption coefficient. For lines one can also write:

$\Phi_{i j}=h v\left[n_{j}\left(A_{j i}+B_{j i} \bar{J}\right)-n_{i} B_{i j} \bar{J}\right]$,

where $n_{j}$ and $n_{i}$ are the upper and lower level densities, respectively, $A_{j i}, B_{j i}$ and $B_{i j}$ are the Einstein coefficients, and $\bar{J}$ is the mean intensity weighted by the line absorption profile and integrated over the line (Avrett 1985).
We used the above formulae to compute NRCR, and Eq. (12) for continua and Eq. (13) for lines. The hydrogen losses are computed inside the main atmosphere code and we consider all transitions within our 5-level plus continuum model atom. Then we add to them the losses computed for a 5-level plus continuum model of Ca II, namely the losses in $\mathrm{Ca}$ II $\mathrm{H}$ and $\mathrm{K}$ lines and in the infrared triplet lines. Other losses are treated as optically thin using the rates of Raymond et al. (1976). In the range of depths where the SXR energy deposit has its maximum, the magnesium losses for the reference model F1 are small (Avrett et al. 1986) and we neglect them in our computations. We also neglect the losses due to the negative hydrogen. These losses can play a role in the lower chromosphere and thus our total losses are somewhat underestimated and we can consider them as a lower limit to real losses. To compare our radiative losses with previously published values, in Fig. 11 we present a comparison between the NRCR calculated by our codes for the VAL-C and F1 semiempirical models and the NRCR calculated by Vernazza et al. (1981) and Avrett et al. (1986) for these models. Our calculations are in good agreement with its.

Finally, we want to make a comment on our treatment of SXR energy deposit. As shown in the previous section, we consider only the absorption of SXR in the transfer equation and this is because we do not expect any SXR emision from the chromosphere itself. In the range of SXR wavelengths, this absorption is covered by a part of the integral in Eq. (12). This part of the integral represents the radiative heating of the plasma by SXR (NRCR is negative). The rest of the integral behind the SXR wavelength range covers all other NRCR which we compute as described above.

\section{A comparison between SXR heating and radiative cooling}

By using our procedures we determined the height distribution of the energy deposited from SXR radiation within the 1-300 spectral range. For calculations of the incident intensities of SXR we used the parameters $E M, T$ and $A$ determined for both flares from Yohkoh SXT images (see Table 1). We have also used the models of the solar chromosphere determined in the 

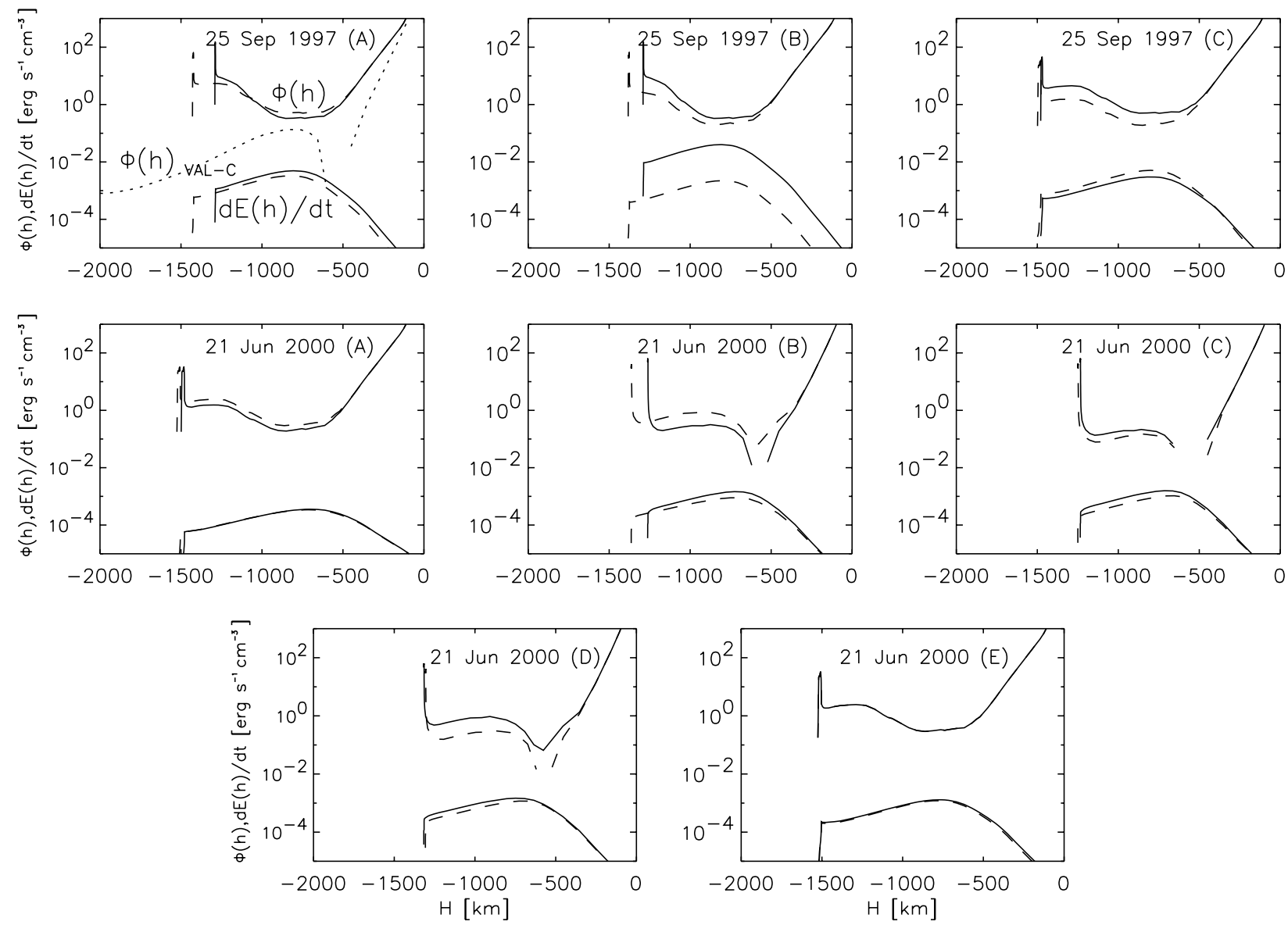

Fig. 12. The energy deposit $\mathrm{d} E(h) / \mathrm{d} t$ in the chromosphere by soft X-ray radiation and the net radiative cooling rates $\Phi(h)$ calculated for the analyzed areas of the two flares. Upper row: plots made for areas A, B and C for the flare of 25 September 1997 at 12:05:52 UT (continuous lines) and 12:18:15 UT (dashed lines). Middle and bottom rows: plots made for areas A, B, C, D and E for the flare of 21 June 2000 at 10:46:08 UT (continuous lines) and 10:50:56 (dashed lines). The net radiative cooling rates $\Phi(h)_{\text {VAL-C }}$ for VAL-C model chromosphere are also plotted in the first graph (dotted line).

analyzed parts of both flares (Sect. 4). In Fig. 12 we present the height distribution of the energy deposit $(\mathrm{d} E(h) / \mathrm{d} t)$ in selected parts of flares by SXR radiation (lower continuous and dashed lines). In these plots we can see that the behaviour of all distributions of the energy deposit is similar with flat maxima of around $750 \mathrm{~km}$ above the photosphere ( $h=0$ corresponds to $\tau_{5000}=1$ ). As was shown in Berlicki (2002), the shape of the curves of deposited energy is similar for different models of the chromosphere. Only the amount of the energy deposited in the upper chromosphere is affected by the existence of the Chromosphere-Corona Transition Region (CCTR). The position of the CCTR is model-dependent and it determines the layer of a sudden drop of the deposited energy with height. In our case the models of the chromosphere determined in all selected areas of flares were not significantly far from the starting models (VAL-C or F1). The temperature of X-ray sources also does not affect significantly the distribution of the deposited energy. In the case of both analyzed flares the range of temperatures of X-ray sources was between 6.2 and 15.0 MK.

Other parameters which influence the height distribution of the deposited energy are the emission measure $E M$ and projected area $A$ of the X-ray source. The ratio of the $E M$ to the projected area $A$ of the source determines the soft X-ray intensity at the top of the chromosphere (see Eq. (10)). The analysis of the influence of the $E M / A$ ratio on the energy deposit performed in Berlicki (2002) showed that the shape of the height distribution of the X-ray energy deposited in the chromosphere is not sensitive to this ratio, but it affects the vertical position of the curves. In other words, the amount of SXR energy deposited increases with increasing $E M$. To illustrate this effect in Fig. 13 we present the X-ray energy deposited in VAL$\mathrm{C}$ and F1 models calculated for two theoretical X-ray sources which have equal temperatures and projected areas but with different emission measures.

In our analysis we assumed that the energy input to the given volume element by different heating processes is equal to the energy loss from this element. This assumption is valid only if the time-scale of radiative processes in the solar atmosphere is much shorter than the thermodynamical time-scale. During late phases of the flares the changes of flare plasma parameters are rather slow and we can consider the evolution of the flare as a series of quasi-static atmospheres in energetic balance. Our analysis was performed for the gradual phase of two solar flares. Therefore, the amount of NRCR in a given layer of 

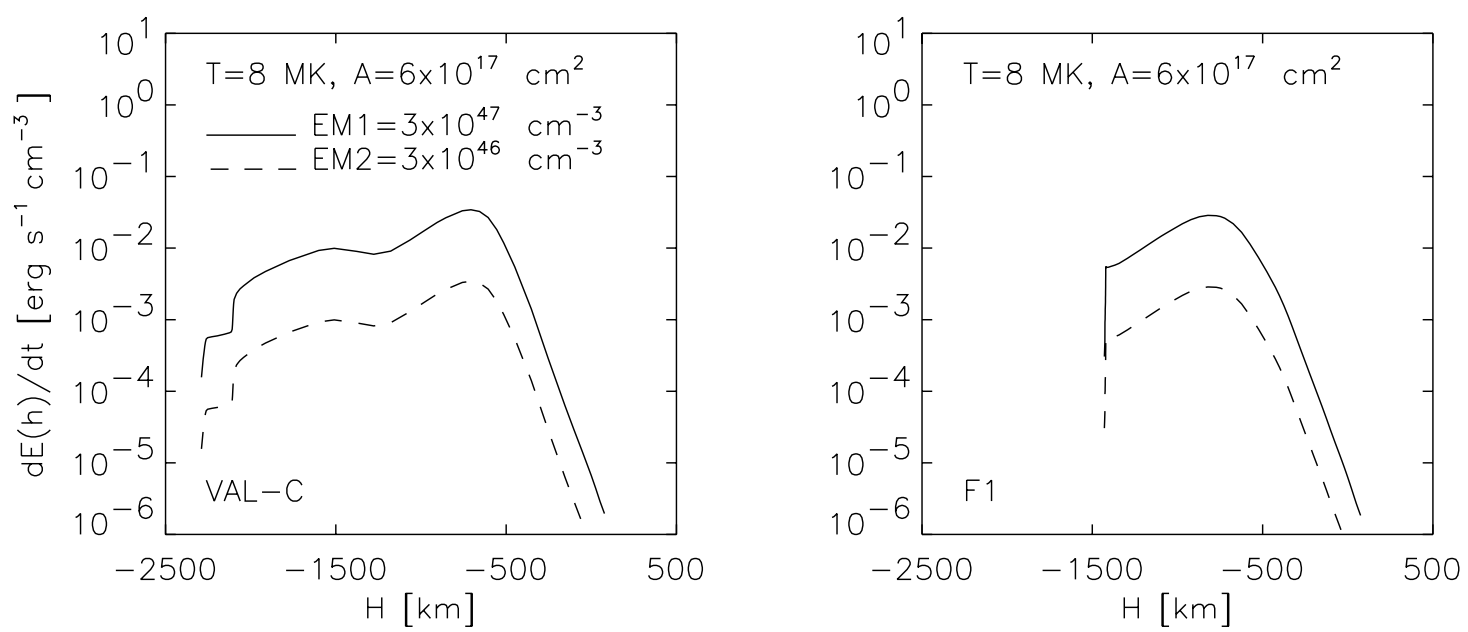

Fig. 13. X-ray energy deposit $(\mathrm{d} E(h) / \mathrm{d} t)$ in semiempirical models VAL-C (left) and F1 (right) calculated for two X-ray sources with equal temperatures $T$ and projected areas $A$ but with different emission measures $\left(E M 1=3.0 \times 10^{47} \mathrm{~cm}^{-3}-\right.$ solid lines, $E M 2=3.0 \times 10^{46} \mathrm{~cm}^{-3}-$ dashed lines).

the solar atmosphere can be considered to be equal to the total energy input in the same layer. If we know how much energy is delivered to such a layer through some specific heating mechanism, we can compare it with the cooling rates and determine the contribution of that heating mechanism to the total energy input.

To compare the SXR energy deposit with radiative losses we present in the upper parts of all graphs in Fig. 12 the plots of the NRCR $(\Phi(h)$, continuous and dashed lines). The calculations of these radiative losses were performed for the models of the chromosphere determined for both flares in all analyzed areas and at all times. Nevertheless, for clarity, for the flare of 25 September 1997 we present in Fig. 12 only the plots of the first (12:05:52 UT) and last (12:18:15 UT) moments of time. All plots of radiative losses have their minima located between about 600 and $800 \mathrm{~km}$ above the photosphere. Around the same heights, the amount of deposited energy has a maximum for all analyzed areas (cf. Fig. 12). However, at all heights in the chromosphere the amount of the energy deposited by SXR radiation is much smaller than the NRCR. From among all analyzed areas the ratio of deposited energy to the NRCR is maximal for an area B of the flare of 25 September 1997. In this case $[\mathrm{d} E(h) / \mathrm{d} t] / \Phi(h) \approx 0.15$ at $h=800 \mathrm{~km}$ above the photosphere.

In the first graph in Fig. 12 we also plotted the radiative losses calculated for the VAL-C model of the QS atmosphere (dotted line). The VAL-C model atmosphere is static and radiative losses give us an idea of how much of the heating we need to achieve the global energy balance in this atmosphere (but see dynamical models of Carlsson \& Stein 1995). We do not consider particular mechanisms of the heating but we can say that the NRCR at each level of the atmosphere should be equal to the energy input to the given level (Vernazza et al. 1981). This kind of heating, if realistic, can be treated as stationary heating present all the time in the QS atmosphere. During the flares, besides this "background" heating, additional heating mechanisms appear in the chromosphere. This suggests that the NRCR calculated for the flaring chromosphere should be equal to the sum of QS atmosphere heating and flare-driven heating mechanisms, although the problem is highly nonlinear. Taking into consideration these remarks and assuming that the background heating is the same during the flare, we could subtract the height distribution of the NRCR calculated for the VAL-C model of the QS atmosphere from the height distribution of the NRCR calculated for the analyzed areas in both flares. The result of this subtraction can be compared with the height distribution of the energy deposited by SXR radiation $(\mathrm{d} E(h) / \mathrm{d} t)$. In Fig. 12 it is clearly seen that for all analyzed areas the amount of energy deposited by SXR radiation is very low in comparison with both $\Phi(h)_{\mathrm{VAL}-\mathrm{C}}$ and $\Phi(h)$ curves. This suggests that the energy provided by SXR radiation to all layers is not sufficient to explain the amount of radiative losses calculated for the analyzed areas of flares. Only in the layers located around $750 \mathrm{~km}$ above the photosphere the SXR energy deposit seem to be sufficient to contribute to the heating.

In our determination of the models in analyzed areas of the flares we used only the $\mathrm{H} \alpha$ line profile. This line is commonly used in the modelling of the solar flares because of its sensitivity to various heating processes (Canfield et al. 1984). The comparison between the energy supplied to the flaring atmosphere by SXR radiation and the calculated NRCR showed that the SXR energy deposit is not sufficient to explain the enhanced $\mathrm{H} \alpha$ emission observed in analyzed areas of flares. This means that there is not enough energy input to the layer of the solar atmosphere where the $\mathrm{H} \alpha$ line is formed. The depths where a spectral line is formed can be described by the contribution function $(\mathrm{CF})$. This function is defined as (see e.g. Kašparová \& Heinzel 2002):

$I_{\nu}(0)=\int C F(h) \mathrm{d} h$

where $I_{v}(0)$ is the emergent specific intensity. Taking into account the form of a formal solution of the equation of radiative transfer for a semi-infinite atmosphere (for normally emergent radiation) and using $\mathrm{d} t_{v}=-\chi_{v} \mathrm{~d} h$ and $S_{v}=\eta_{v} / \chi_{v}$, we have

$C F=\eta_{v}(h) \mathrm{e}^{-t_{v}(h)}\left[\operatorname{erg~cm} \mathrm{cm}^{-3} \mathrm{~s}^{-1} \mathrm{sr}^{-1} \mathrm{~Hz}^{-1}\right]$, 


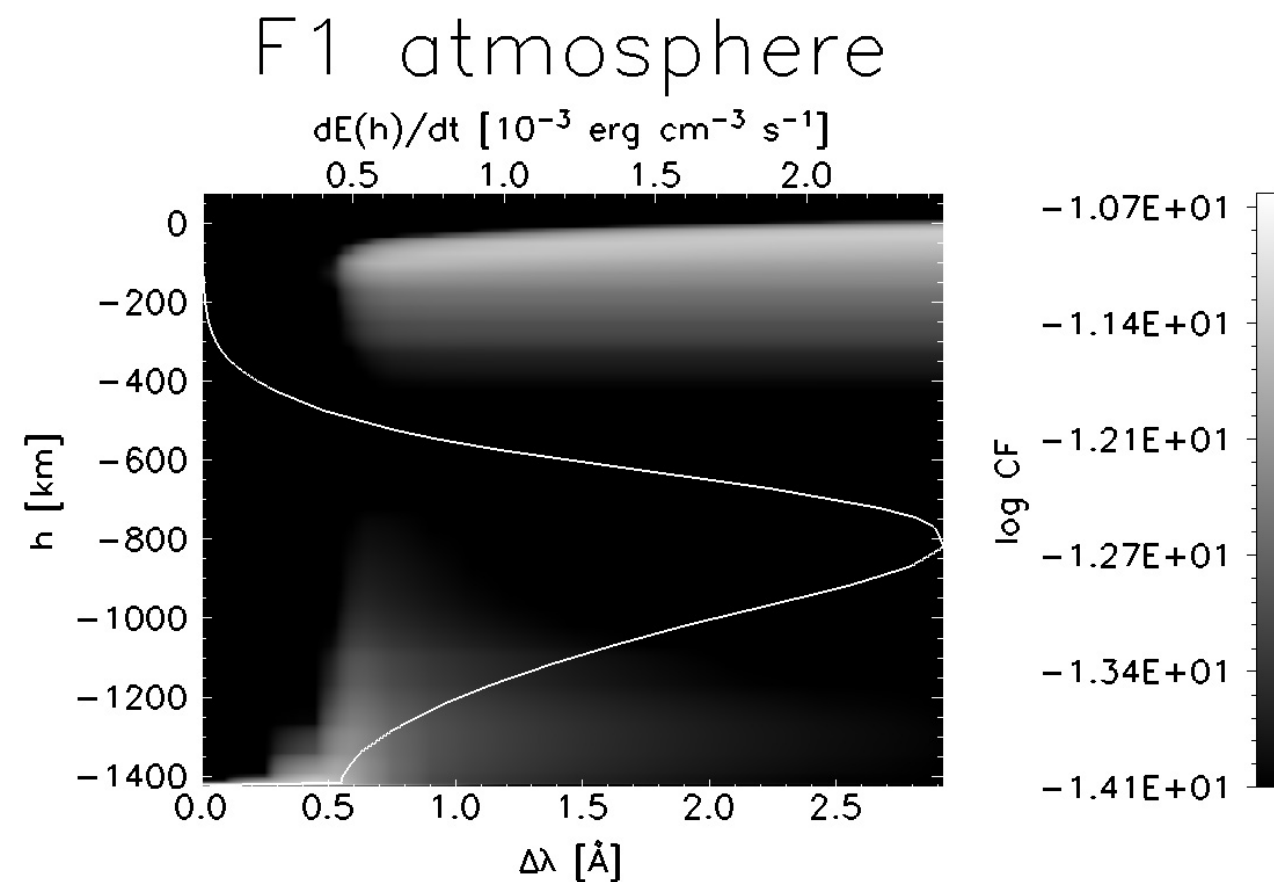

Fig. 14. $\mathrm{H} \alpha$ contribution function as a 2D function of the wavelength from the line centre and the height in the atmosphere for the flare model obtained for area A at 12:09:25 UT for the flare of 25 September 1997. The height distribution of the SXR energy deposit rate $\mathrm{d} E(h) / \mathrm{d} t$ is over-plotted (white line).

where $S_{v}$ is the line source function, $\eta_{v}$ is the emission coefficient, $\chi_{v}$ is the absorption coefficient, and $t_{v}$ is the optical depth. Calculations of the contribution function performed for the $\mathrm{H} \alpha$ line formed in a F1 weak-flare model atmosphere showed that the formation of the line core between $\pm 0.3 \AA$ occurs high in the chromosphere close to the CCTR (Fig. 14). In this layer the amount of deposited energy is much lower in comparison to its maximum value at $h \approx 750 \mathrm{~km}$.

Our semiempirical models are the modifications of F1 or VAL-C atmospheres and were constructed to give synthetic $\mathrm{H} \alpha$ profiles compatible with flare observations. Therefore, the most significant modifications should correspond to regions where $\mathrm{H} \alpha$ is formed. In the MSDP wavelength range it is the middle and upper chromosphere (Fig. 14). The lower chromosphere and temperature-minimum region do not play a significant role in the $\mathrm{H} \alpha$ line formation because of very low hydrogen excitation (low temperatures). The temperature structure of our models and the corresponding NRCR would be somewhat different if other spectral lines and continua were used for model construction. But fortunately, as we have demonstrated, SXR energy deposit is not very sensitive to details of the model but depends critically on the $E M$ of the SXR source. Moreover, our results for two flares observed at different positions and different times seem to be robust enough (i.e. a large difference between SXR heating and NRCR) so that small modifications of the models will not change their general character.

\section{Conclusions}

In this paper we have studied the energy budget of a solar flaring chromosphere irradiated by soft X-rays coming from overlying hot flare loops. To analyze the expected effect of the X-ray heating we used the observations of two solar flares of 25 September 1997 and 21 June 2000. These flares were observed in the $\mathrm{H} \alpha$ line during the gradual phase with the Wrocław University Multichannel Subtractive Double Pass spectrograph coupled to the Large Coronagraph. We also used the X-ray observations of flares taken with the Yohkoh Soft $\mathrm{X}$-ray Telescope. Using these observations together with methods of radiative transfer and non-LTE modelling, we were able to analyze the relation between the amount of energy deposited in the solar atmosphere by SXR radiation and the net radiative cooling in the atmosphere. We have made, for the first time, detailed computations of the energy deposit in the solar atmosphere by SXR radiation, using simultaneous $\mathrm{H} \alpha$ and SXR observations of flares. The $\mathrm{H} \alpha$ observations and constructed grid of the solar atmosphere models permitted us to determine the structure of the atmosphere in the analyzed areas of the flares and to calculate the height distribution of the NRCR. The SXR observations were used to find the amount of the energy supplied to the solar atmosphere by photoionization processes.

Our analysis shows that the energy deposited in the analyzed areas of two solar flares by SXR radiation within the $1-300 \AA$ spectral range is typically much lower than the radiative losses calculated in these areas. It is obvious that the heating of the chromosphere by SXR takes place during solar flares but the amount of supplied energy is too low to explain the prolonged emission always observed in chromospheric spectral lines during the gradual phases solar flares. In this work we used flares that had rather low X-ray emission. Thus, the soft X-ray flux reaching the chromosphere was also low. There is a possibility that for another flares the X-ray sources may 
heat some part of the chrompsphere much more efficiently. In our codes which calculate the energy deposited in the chromosphere by SXR we used the ionization cross-sections for different elements taken from Brown \& Gould (1970). There is the possibility that this data could differ from more recent values. Nevertheless, the possible differences should not influence the obtained results.

During the impulsive phase of solar flares, chromospheric emission can be explained as a result of non-thermal heating of the chromosphere by electron beam bombardment (Brown 1973; Emslie 1978). In the gradual phase non-thermal heating seems to be negligible. Therefore, we need other sources of energy. Thermal conduction is effective only in the layers of the atmosphere where the temperature gradient is high enough. This means that this mechanism cannot supply much energy to the middle and lower chromosphere (Ricchiazzi \& Canfield 1983). Nevertheless, in solar flares the central part of the $\mathrm{H} \alpha$ line profile is formed high in the chromosphere close to a steep temperature jump (cf. Fig. 14 in Sect. 6). Thus, in this part of the chromosphere heating of the plasma may be more likely due to thermal conduction as suggested by Czaykowska et al. (2001). In any case, the conductive heating will cause the so-called "gentle evaporation" of the upper chromosphere and transition region (Schmieder et al. 1987; Schmieder et al. 1990; Czaykowska et al. 1999). The increased coronal pressure inside the hot loops overlying the $\mathrm{H} \alpha$ kernels will then lead to enhancement of the $\mathrm{H} \alpha$ line emission as shown by Canfield et al. (1984). This is consistent with our semiempirical modeling where we increased the column mass by $m_{0}$ to simulate this mechanism. The process of a gentle evaporation during the gradual phase of a flare is now under study, using multiwavelength data for flare of 22 October 2002 (Berlicki et al. in preparation).

Acknowledgements. The authors thank the Yohkoh Team and the YDAC at Mullard Space Science Lab. for the SXT data and the SoHO/MDI consortium for the MDI magnetogram data. SoHO is a joint project of ESA and NASA. This research was partially supported by the European Commission through the RTN programme (European Solar Magnetism Network, contract HPRN-CT-2002-00313. AB is a member of the European Solar Magnetism Network (ESMN). This work was also partially supported by the grant A3003203 of the Academy of Sciences of the Czech Republic. The authors are indebted to J. Jakimiec, B. Rompolt, J. Sylwester and P. Rudawy for useful discussions and comments.

\section{References}

Avrett, E. H. 1985, in Chromospheric Diagnostics and Modelling, NSO, 67

Avrett, E. H., Machado, M. E., \& Kurucz, R. L. 1986, in The Lower Atmosphere in Solar Flares, NSO, 216

Berlicki, A. 2002, Ph.D. Thesis, Wrocław University, Poland

Brown, J. C. 1973, Sol. Phys., 31, 143

Brown, R. L., \& Gould, R. J. 1970, Phys. Rev. D, 8, 2252

Canfield, R. C., Gunkler, T. A., \& Ricchiazzi, P. J. 1984, ApJ, 282, 296

Carlsson, M., \& Stein, R. F. 1995, ApJ, 440, L29

Cox, D. P., \& Tucker, W. H. 1969, ApJ, 157, 1157

Czaykowska, A., De Pontieu, B., Alexander, D., \& Rank, G. 1999, ApJ, 521, L75

Czaykowska, A., Alexander, D., \& De Pontieu, B. 2001, ApJ, 552, 849

Emslie, A. G. 1978, ApJ, 224, 241

Falewicz, R., \& Rudawy, P. 1999, A\&A, 344, 981

Fang, C., \& Hénoux, J. C. 1983, A\&A, 118, 139

Hawley, S. L., \& Fisher, G. H. 1992, ApJS, 78, 565

Heinzel, P. 1995, A\&A, 299, 563

Hénoux, J. C., \& Nakagawa, Y. 1977, A\&A, 57, 105

Jefferies, J. T. 1957, MNRAS, 117, 493

Kašparová, J., \& Heinzel, P. 2002, A\&A, 382, 688

Machado, M. E. 1978, Sol. Phys., 60, 341

Machado, M. E., Avrett, E. H., Vernazza, J. E., \& Noyes, R. W. 1980, ApJ, 242, 336

Mein, P. 1991, A\&A, 248, 669

Mewe, R., Gronenschild, E. H. B. M., \& van den Oord, G. H. J. 1985, A\&AS, 62, 197

Mewe, R., Lemen, J. R., \& van den Oord, G. H. J. 1986, A\&AS, 65, 511

Raymond, J. C., Cox, D. P., \& Smith, B. W. 1976, ApJ, 204, 290

Raymond, J. C., \& Smith, B. W. 1977, ApJS, 35, 419

Ricchazzi, P., \& Canfield, R. C. 1983, ApJ, 272, 739

Rompolt, B., Mein, P., Mein, N., Rudawy P., \& Berlicki, A. 1993, JOSO Annual Report, 87

Rosner, R., Tucker, W. H., \& Vaiana, G. S. 1978, ApJ, 220, 643

Rybicki, G. B., \& Hummer, D. 1991, A\&A, 245, 171

Schmieder, B., Forbes, T. G., Malherbe, J. M., \& Machado, M. E. 1987, ApJ, 317, 956

Schmieder, B., Malherbe, J. M., Simnett, G. M., Forbes, T. G., \& Tandberg-Hanssen, E. 1990, ApJ, 356, 720

Somov, B. 1975, Sol. Phys., 42, 235

Švestka, Z. 1957, Publ. Astron. Inst. Prague, 32

Tsuneta, S., Acton, L., Bruner, M., et al. 1991, Sol. Phys., 136, 37

Vernazza, J. E., Avrett, E. H., \& Loeser, R. 1981, ApJS, 45, 635 